\title{
Theoretical and Therapeutic Applications of Mental Pain in Childhood cancer Survivors of the Findings of the paper
}

\author{
Shulamith Kreitler ${ }^{1}$, Haya $\operatorname{Raz}^{2^{*}}$ \\ ${ }^{1}$ School of Psychological Sciences Tel-Aviv University and Sheba Medical Center, Tel-Hashomer Psycho-oncology Research Center. \\ ${ }^{2}$ Head of Nursing School, Faculty for Life Sciences and Health, Jerusalem College of Technology, Israel.
}

*Corresponding Author : Haya Raz, Head of Nursing School, Faculty for Life Sciences and Health, Jerusalem College of Technology, Israel. E-mail: hayar@jct.ac.il

Received date: November 23,2018;Accepted date : December 03,2018; Published date: December 05,2018.

Citation: Haya Raz, Shulamith Kreitler. Theoretical and Therapeutic Applications of Mental Pain in Childhood cancer Survivors of the Findings of the paper, J. Psychology and Mental Health Care . Doi: http://dx.doi.org/ 10.31579/ 2637-8892.18/007.

Copyright : (c) 2018 Haya Raz .This is an open-access article distributed under the terms of The Creative Commons Attribution License, which permits unrestricted use, distribution, and reproduction in any medium, provided the original author and source are credited.

\begin{abstract}
The paper deals with a review of "Mental Pain in Israeli Adult Childhood Cancer Survivors and Its Effects on their Quality of Life" by H. Raz, N. Tabak, Y. Alkalay, \& S. Kreitler (1). The review focuses on theoretical and therapeutic implications of the findings. The major theoretical elaboration of the findings focuses on the construct of mental pain. This seems advisable both because there are several apparently similar concepts which presumably render mental pain superfluous and because mental pain was found to characterize pediatric cancer survivors who otherwise were found to be well adjusted as adults. The new redefinition of mental pain focuses on the components of low self-image and low meaningfulness of life. The constructs of mental pain in the past and of tolerance of mental pain were found to be less useful in this context. The new redefinition points to two new venues of interventions for reducing mental pain: enhancing self-image and increasing meaningfulness of life. The two kinds of interventions, which are based on elaborating meanings, are briefly described.
\end{abstract}

Keywords: mental pain; meaning; pediatric cancer; survivors; meaningfulness of life.

\section{Introduction}

The reviewed paper focuses on survivors of pediatric cancer. It examines recovered individuals who are at present young adults and about 13 years earlier have been diagnosed and treated successfully for cancer. The reported study deals with their mental pain and its impact on their quality of life. The results show that the mental pain of the participants in the present as well as that recalled from the past is high and lowers significantly their present quality of life.

The purpose of the present review is to elaborate some of the major aspects of the study from theoretical and applied perspectives.

\section{Theoretical Implications}

The focus of the paper is mental pain. Mental pain is a new construct that did not yet get established in the arena of the behavioral sciences in general and in pediatric psycho- oncology in particular. One reason may be that it deals with the negative aspects of the internal atmosphere of individuals. This domain seems to be already overcrowded with constructs, such as depression (with its different kinds e.g., clinical, neurotic, psychotic), tension, stress, suffering, demoralization, sadness, melancholia, despair, distress, existential distress, misery, sorrow, unhappiness, dysthymia, sorrow, sadness, hopelessness, bleakness, despondency. In view of this plethora of designations of the negative emotional experiences, it is justified and necessary to inquire about the specific contribution of "mental pain".

A review of the definitions and correlates of mental pain, most of which are presented in the paper itself, highlights several features that differentiate mental pain from other affiliated constructs. Major among these features are the sense of low self-image and the sense of meaninglessness. Each of these characteristics may be due to a variety of causes. For example, meaninglessness reflects a sense of emptiness [2] and may arise because of disappointments, for example, in the domains of marital life, love relationship, parenthood, career, or financial situation [3,4]. Low self-image, defined also as selfawareness of inadequacy or self-disappointment [5], may be due to a discrepancy between one's self-image and ideal image [6] or between one's low achievements and high aspirations [5].
It could be triggered by losing a beloved person, or by being rejected by a partner $[7,8]$, or through frustration of one's basic needs, such as to have control, to protect one's self-image, and to feel secure [9]. These two basic characteristics may be separate or together, may develop each by itself or get intertwined with one another. For example, one may feel one's life is meaningless but that one is strong and able enough to reconstruct the meaningfulness; or, inversely, one may feel that one's life has meaning but that one is too weak or worthless psychologically or physically to implement the operations that could substantiate the meaningfulness of one's life. On the other hand, it is possible that both characteristics are intertwined from the very beginning, as for example, when one feels worthless because one's life is meaningless or that one feels one's life is meaningless precisely because one's self and personality are worthless.

The co-activation of both characteristics may be responsible for the deep and pervasive experience accompanying the evocation of mental pain. It is often described as reflecting dissatisfaction with oneself on the spiritual and existential levels of being, since one does not fulfill one's obligations as a human being [9]

There are several implications to these conceptualization of mental pain. One is that mental pain is a universal human condition, so that every human being is in principle liable to develop mental pain to some extent. Secondly, mental pain is not to be considered necessarily as a psychopathological symptom because it is rooted in existential characteristics of being human, which are the striving for meaningfulness and for self-realization. Thirdly, mental pain is a highly unpleasant experience which one would like to set aside or terminate. It is described as intense psychological pain [8], perturbation, inner turmoil, feeling upset, mental disturbance [9], unbearable mental suffering and extremely aversive feeling which can be experienced as torment [10]. It is such a difficult emotion to contain or overcome that tolerance of mental pain is being considered as a separate ability of the individual reflecting one's capacity to cope with mental pain.

The extreme unpleasantness of the experience is reflected in the nine scales that make up the questionnaire of mental pain [11]: Loss of control: e.g. "I lack of control over what is happening inside me"; Irreversibility of pain:e.g."Something in my life changed forever"; Emotional flooding: e.g. 
"There is a storm of emotions in me"; Narcissistic wounds: e.g. "No one is interested in me"; Estrangement: e.g. "It is as if I was not real"; Confusion: e.g. "I cannot concentrate"; Need for social support: e.g. "I need support from my surroundings"; Emptiness: e.g. "I have no desire for anything"; Freezing: e.g. "It's like I'm paralyzed".

The intense suffering characterizing mental pain motivates the desire to get away from that experience, terminate it and free oneself from it. This motivation may result in enhanced efforts to generate meaningfulness as a human being, for doing something significant for realizing one's goals and desires by using the potentials in one's self and personality. The outcome may be described as similar to posttraumatic growth [12,13]. This conclusion is supported by the evidence that post-traumatic growth is based primarily on finding meanings which enable reconstructing one's meaningfulness of life [14].

Another outcome of the urge to terminate mental pain may be suicide. Mental pain is a quite reliable predictor of suicide attempts or acts, even better than depression [15-18]. Hence, suicide has been considered as an escape from mental pain [8,10,19-20]. Again, in view of the suffering involved in mental pain, suicide triggered by mental pain is not to be considered necessarily as a psychiatric or psychopathological act.

The suffering characterizing mental pain is reflected in the present study in the impact mental pain has on reducing quality of life. It is of special interest to note that the mental pain scales that played the most prominent role in regard to quality of life were narcissistic wounds (significant predictor in 8 cases) and emptiness (in 5 cases). It is clear that narcissistic wounds refer to the self-image and emptiness to meaninglessness. These two scales of mental pain are the salient components of mental pain as elaborated in the present review.

There are two main advantages to the proposal to define mental pain in terms of the two foci of low self-image and low meaningfulness of life. First, the redefinition provides a clarification of the specific characteristics of mental pain, which may justify the preservation of this construct in an already overcrowded arena of negatively oriented constructs. It enables, on the one hand, a clearer distinction between mental pain and the other apparently similar constructs, such as depression, dysthymia and suffering in general, and, on the other hand, it provides salience to the bridge between mental pain and the existentialist approach. Although low self-image and meaninglessness may play a role, for example, also in depression or existential distress, they are not in these frameworks the typical distinguishing features as in regard to mental pain. Both implications provide the possibility of carving for mental pain a specific space in the behavioral sciences, with a potential contribution to understanding both the negative and positive reactions to mental pain.

Secondly, the redefinition of mental pain highlights specific interventions for mitigating mental pain grounded in the particular defining characteristics (see Therapeutic Implications).

The two major conclusions concerning the theoretical approach to mental pain may be complemented by several further specifications, based on the findings of the study. First, it appears that there is no special advantage to focusing separately on the nine scales making up the score of mental pain. One reason is that most of these scales do not shed light on any particular aspects of mental pain beyond emphasizing different aspects of the suffering itself. A second reason is that most of the scales are interrelated positively and significantly, which means that they actually assess the same construct.

Another conclusion supported by the findings concern the time frame for the assessment of mental pain. In the original study subjects were requested to assess their mental pain in the present and in the past, separately. The findings indicate that mental pain in the past, as reported in the present, constitutes largely a part of mental pain in the present, since it is correlated positively with mental pain in the present and its effects are overshadowed by those of mental pain in the present.
The scores of mental pain in the present reflect probably mental pain in the past, in the original situation when the diagnosis and treatments took place, as well as additional painful experiences in the aftermath. However, it is difficult for the person to differentiate between mental pain in the present and in the past and to recall adequately the emotional experiences from the past. Hence, for the sake of theoretical clarity it is best to focus on the assessment of mental pain in the present rather than in the past.

A second conclusion concerns tolerance of mental pain. Its three constituting scales - congestion with coping, congestion with containment, and coping with containment - are intercorrelated but are not correlated directly with quality of life. The impact of tolerance of mental pain seems to be limited to specific aspects of quality of life and to be mediated primarily through mental pain in the present. Further, the items do not provide information about the bases of mental pain tolerance, which could be the outcome of personality traits or of coping processes. Be it as it may, the items do not provide information about coping with mental pain. Hence, it seems that there is no theoretical necessity to consider mental pain tolerance as a component or accompaniment of mental pain.

\section{Therapeutic Implications}

The major findings of the study were that survivors of pediatric cancer score high on mental pain and that mental pain has a pervasive negative impact on the survivors' quality of life. In view of the findings in many studies that survivors as adults are rather well-adjusted in their daily life, it seems that their high scores in mental pain constitute one of the few indicators that not all is well in the domain of pediatric cancer survivors. This conclusion implies that pediatric cancer survivors are in need of help that could provide tools for coping with mental pain and for mitigating it.

The proposal to define mental pain primarily in terms of low self-image and low meaningfulness of life indicates major venues for intervention in regard to mental pain. One focuses on the self-image, the other on the meaningfulness of life, represented in the mental pain scale in terms of the scales of narcissistic wound and emptiness, respectively. Both tracks may be applied, singly or in combination.

The "self-image intervention" consists of two main phases. Both phases are based on the prior assessment of the meaning-based self-image which presents one's self-image in terms of the different meaning dimensions, e.g., one's actions feelings, manner of functioning [21]. The first phases deals with enriching one's self-image. This includes mainly enhancing one's awareness of the abilities and features one already has but is not aware of. The other deals with expanding one's self-image by developing abilities and features that are weak but could potentially be enhanced. Both steps are implemented by focusing on aspects of the self in theoretical terms (e.g., what does feature X mean?), in operational terms (e.., in which manner feature $X$ functions), and through examples (e.g., please give examples of feature $\mathrm{X}$ in your life/behavior). This tribrachic training approach is applied separately in regard to each aspect or facet of the self that needs to undergo strengthening of awareness or activation. Sometimes it may be necessary to apply the second phase of the "selfimage intervention" which consists in reducing the gap between the present self-image and the aspired one. This phase is necessary when the aspired self-image is out-of-bounds for the individuals in terms of the aspired contents or the levels. In this case the training consist in making the individual aware of the gaps between the present and aspired selfimage in terms of each of the involved facets of the self.

The "meaningfulness of life" intervention is based on the conception that the meaningfulness of life consists of meaning aspects that belong to four clusters: factional-dynamic, sensory-perceptual, experiential-cognitive and contextual [3, 14, 22]. The training consists in an on-line procedure in which the individual is presented with items from the four groups and requested to select in each group one item. In regard to the selected item, the subject was asked to check Yes or No for each of 3 stated reasons for the choice, and to answer questions, such as "Have you ever done something that exemplifies this item or has it ever happened in your life in some form? Yes/no". The beneficial results of the training were shown in regard to the meaningfulness of life itself which is enhanced and in regard to increasing one's quality of life and reducing one's existential distress [22]. The latter may be considered as resembling mental pain. 


\section{Conclusions}

The review supports the conclusion that mental pain is a valuable construct with an important contribution to the field of cancer survivorship. It has a unique set of contents defined by characteristics that are overlooked or overshadowed by other characteristics in the context of apparently similar constructs, ranging from depression to distress. The unique parameters defining mental pain focus on the self and on the meaningfulness of life. Mental pain results when either one or both are challenged and lose their contents or volume. The special characteristic of mental pain is that it indicates that something essential is missing or is not quite as expected in regard to one's self and one's meaning in life. Highlighting these two facets makes it potentially possible to apply adequate interventions to correct the specific domains in one's life. Thereby the clarification in the present review provides the means for shaping new tools for alleviating the pain of living in cancer survivors.

\section{References}

1. H. Raz, N. Tabak, Y. Alkalay, \& S. Kreitler (2016). Mental Pain in Israeli Adult Childhood Cancer Survivors and Its Effects on their Quality of Life. Journal of Psychology and Psychotherapy, $6(2)$.
2. Frankl VE (1963). Man's search for meaning: An introduction to logotherapy. Pocket Books, New York.

3. Shneidman ES (1993) Suicide as psychache. Journal of Nervous \& Mental Disease 181: 147-149.

4. Kreitler S (2016). Meanings of meaningfulness of life. In A. Batthyany (Ed.), Logotherapy and existential analysis (Vol. 1). Springer: Vienna, Austria, pp. 95-106.

5. Baumeister RF (1990) Suicide as escape from self. Psychol Rev 97: 90-113.

6. Joffe WG, Sandler J (1967) On the concept of pain, with special reference to depression and psychogenic pain. J Psychosom Res 11: 69-75.

7. Freud S (1959) Inhibition, symptoms and anxiety. In: J Strachey (Ed.), The standard edition of the complete psychological works of Sigmund Freud. Hogarth Press, London. 